\title{
A meso elastoplastic constitutive model for polycrystalline metals based on equivalent slip systems with latent hardening ${ }^{*}$
}

\author{
LIANG Naigang (梁乃刚), LIU Honggiu（刘洪秋) \\ and WANG Ziqiang (Tzuchiang WANG，王自强) \\ (Institute of Mechanics, Chinese Academy of Sciences, Beijing 100080, China)
}

Received November 31, 1997

\begin{abstract}
A meso material model for polycrystalline metals is proposed, in which the tiny slip systems distributing randomly between crystal slices in micro-grains or on grain boundaries are replaced by macro equivalent slip systems determined by the work-conjugate principle. The elastoplastic constitutive equation of this model is formulated for the active hardening, latent hardening and Bauschinger effect to predict macro elastoplastic stress-strain responses of polycrystalline metals under complex loading conditions. The influence of the material property parameters on size and shape of the subsequent yield surfaces is numerically investigated to demonstrate the fundamental features of the proposed material model. The derived constitutive equation is proved accurate and efficient in numerical analysis. Compared with the self-consistent theories with crystal grains as their basic components, the present theory is much simpler in mathematical treatment.
\end{abstract}

Keywords: elastoplastic, poly crystalline, latent hardening.

With the development of modern microscope-observation and computer techniques in the study of mechanical properties of material, plasticity theories based on physical mechanisms have made great progress. A systematic survey of the rigorous investigation of an individual crystal grain and its macroscopic response as well as the approximate polycrystal models was given by Havner ${ }^{[1]}$. Different from the phenomenological ones, the physical plasticity theories are based on the microstructure and mechanical property of crystal grains, so neither potential function of plasticity nor evolution equation of the thermodynamic intrinsic variables is required. They are believed to be the most persuasive plasticity theories.

However, the available physical plasticity theories are not quite satisfactory in practical uses. First of all, the hardening rule of the single crystal under complex loading conditions is still open and a suitable averaging technique is also required to calculate the macro stress-strain from the complicated local stress-strain field in a macro material element including a great number of microcomponents and unpredictable defects. Secondly, observations with modern scanning and transparent electronic microscopes show that the actual micro-structures of polycrystalline material, especially under finite deformation, are far from those of the existing micro-mechanical material models. Sliding takes place not only in crystal grains but also on their boundaries. The latter even plays a major role in some cases. In crystal grains, active slip planes are usually apart from each other at some distance, so the plastic strain is formed by the relative motion of crystal slices. The microstructure changes with grain breakage, recrystallization, defect formation, , boundary evolution, phase transformation, damage and so on. It will result in tremendous mathematical

* Project supported by the National Natural Science Foundation of China (Grant No. 19732006). 
complexity by taking account of the nonuniformity of the local stress-strain field in polycrystalline metals.

Nevertheless, some of the complicated interactions of the micro ingredients in a macro material element have little effects on its macro mechanical behavior. Using a 3-slip-system model, Tokuta et al. ${ }^{[2]}$ successfully predicted the stress-strain responses of metals under complex loading conditions. They claimed that either FCC or BCC crystals can be well simulated by such a material model. Analogous simplification can be traced back to the 1950 s. Batdorf and Budiansky ${ }^{[3]}$ proposed a simple slip model, in which each grain has only one slip system. The well-known experimental evidence is that the earliest-appearing slip lines in polycrystalline metals are always parallel to the plane with the largest shear stress exerted, and the critical resolved shear stress is almost the same under different loading conditions. Therefore, in the isotropic polycrystalline aggregate, a close approximation to the real behavior can be obtained by assuming that slip can occur along all planes in a homogeneously loaded element ${ }^{[4]}$, and the stress-uniform assumption seems acceptable when a material element is sufficiently larger than its constitutional grains. In other words, the actual tiny slip systems can be replaced with some equivalent macro slip systems. Once the hardening rule of the macro slip systems is formulated a constitutive equation can be derived to predict the macro stress-strain responses. In this way, Liang and Cheng ${ }^{[5]}$ proposed a material model for metals with strong grain boundaries and at relatively low temperature. In this paper, we will concentrate on active hardening, latent hardening and Bauschinger effects as well as their influence on size and shape of the subsequent yield surfaces, but the finite deformation effect will be ignored.

\section{Equivalent slip systems and their sliding rates}

It is widely accepted that the plastic deformation mechanism of polycrystalline metals is sliding between the crystal slices in micro-grains and on grain boundaries. Depending on the surroundings, each tiny slip plane has an easy sliding direction and forms a micro slip system oriented randomly in the 3-D space. When activated, the micro slip systems dissipate plastic work and cause plastic deformation. The local stress is actually not uniform in microscopy, but a comparison between the theoretical predictions and the experiments demonstrates that the stress-uniform assumption is reasonable for a material model to simulate macro stress-strain responses. In this case, the driving stress on the planes perpendicular to unit normal vector $\boldsymbol{n}$ along a unit vector $m$ should, on the average, equal the resolved shear stress

$$
\tau=m \otimes n: T=P: T,
$$

where $\boldsymbol{T}$ is the Cauchy stress and

$$
P=\frac{1}{2}(m \otimes n+n \otimes m)
$$

is the orientation tensor. For a slip plane with area $s$ and sliding velocity $v$, the sliding work rate will be $\tau^{\cdot} s^{\cdot} v$.

Now consider the tiny slip systems with normal vectors within a spherical angle $\Delta \Omega$ in direction $n$ and sliding directions within a plane angle $\Delta \Psi$ in direction $\boldsymbol{m}$ in a material element with a volume $V$. Let $s_{i} \Delta \Omega \Delta \Psi$ denote the sliding area with a sliding velocity $v_{i}$. The sliding work rate will be

$$
\Delta \dot{w}=\sum_{i}^{\top} v_{i}\left(\tau s_{i} \Delta \Omega \Delta \Psi\right)=\sum_{i}\left(v_{i} s_{i}\right) \boldsymbol{P}: \boldsymbol{T} \Delta \Omega \Delta \Psi=V \dot{\gamma} \boldsymbol{P}: \boldsymbol{T} \Delta \Omega \Delta \Psi,
$$


where

$$
\dot{\gamma}=\frac{1}{V} \sum_{i} v_{i} s_{i}=\sum_{i} \frac{v_{i}}{l} \cdot \frac{l s_{i}}{V} .
$$

Eq. (4) reveals the physical meaning of $\dot{\gamma}$. If a tiny slip plane is taken as the cross-section of a prism with a height $l, l \cdot s_{i}$ will be the volume of the prism. Let $v_{i}$ denote the relative sliding velocity of the two end surfaces. $v_{i} / l$ will be the average shear strain rate in the prism and $l s_{i} / V$, the volume ratio of the prism to the material element. Therefore, $\dot{\gamma}$ is a statistical average of the sliding rate of the micro slip systems with normal vector $\boldsymbol{n}$ and sliding direction $\boldsymbol{m} . \dot{\gamma}$ is also work-conjugate with the resolved shear stress on the imaginary macro-slip planes perpendicular to $\boldsymbol{n}$. As equivalent energy-consumers, the imaginary slip systems homogeneously-distributing in the 3-D space are taken as the basic components of the present material model.

Thus, the total plastic work rate dissipated in a unit volume is

$$
\dot{\boldsymbol{w}}=\frac{1}{V}\left[\int_{\Omega} \int_{\Psi} \sum_{i}\left(\tilde{v}_{i} \tilde{s}_{i}\right) \tilde{\boldsymbol{P}} \mathrm{d} \Omega \mathrm{d} \Psi\right]: \boldsymbol{T}=\left\{\int_{\Omega} \int_{\Psi} \dot{\gamma} \boldsymbol{P} \mathrm{d} \Omega \mathrm{d} \Psi\right\}: \boldsymbol{T} .
$$

Plastic work rate $\dot{w}$ in eq. (5) should equal the inner product of the plastic strain rate and the stress tensors, $D^{p}: T$. Comparing $D^{p}: T$ with eq. (5) gives

$$
D^{p}=\int_{\Omega} \int_{\Psi} \dot{\gamma} \boldsymbol{P} \mathrm{d} \Omega \mathrm{d} \Psi \text {. }
$$

\section{Constitutive equation}

A slip system cannot be activated if its resolved shear stress $\tau$ is between the critical sliding strength $\tau$, cr and $\tau$ - cr corresponding to its positive sliding direction and the reverse, respectively. That is to say, it must be inactive as long as the stress point is within a range bounded by two hyperplanes defined by $\boldsymbol{P}: \boldsymbol{T}=\tau_{ \pm \mathrm{cr}}$ in the stress space. Hence, the internal envelope hypersurface of the hyperplanes determined by all of the slip systems will be the yield surface, and we can infer the evolution pattern of latent hardening from the measured subsequent yield surfaces if the proportional limits are taken as the yield points. Experiments demonstrate that $\tau_{ \pm \mathrm{cr}}$ of a slip system change during plastic deformation, regardless of its activation state. This is the latent hardening phenomenon. A slip system will be activated if $\tau$ reaches the critical sliding strength and $\dot{\tau}$ is large enough to overcome the latent hardening rate $\dot{\tau}_{\text {latent }}$.

Micro-mechanism of the latent hardening is complicated. $\dot{\tau}_{\text {latent }}$ depends on the orientation, sliding history and activation state of the slip systems. When the crystal material is treated as a homogenized continuum, however, the overall plastic strain rate $D^{\mathfrak{p}}$ is a total representative of the sliding effect of all activated slip systems on each slip system in the homogeneous strain rate field, no mater how it is formed. That is to say, instead of considering the interactions separately for each pair of slip systems, we take latent hardening as an effect of $D^{\mathrm{p}}$ on each of the slip systems in the present work.

Experiments of Phillips and Tang ${ }^{[6]}$ and others demonstrate that the subsequent yield surfaces possess three main characteristics: (i) evident Bauschinger effect-the yield surfaces move with their shape deforming during prestress; (ii) little cross-effect - the size of the subsequent yield surfaces in the direction perpendicular to the prestress path remains almost unchanged; (iii) evident vertex effect— the curvature of the subsequent yield surfaces at the prestress point becomes larger, but the opposite part gets flatter. Lin and Ito ${ }^{[7]}$ predicted the first and the third 
characteristics, but the cross-effect was overestimated.

The orientation tensor $\mathbf{P}$ and the plastic strain rate tensor $\boldsymbol{D}^{\mathrm{p}}$ are vectors in the $6-D$ stressstrain space. The above-mentioned experiments indicate that latent hardening depends strongly on the angle between $\boldsymbol{P}$ and $\boldsymbol{D}^{\mathrm{p}}$. A reasonable assumption is that $\dot{\tau}_{\text {latent }}$ of a slip system is proportional to the scalar product of $\boldsymbol{P}$ and $\boldsymbol{D}^{\mathrm{p}}$, i.e.

$$
t_{\text {latent }}=\chi \boldsymbol{P}: \boldsymbol{D}^{\mathrm{p}}
$$

where $\chi$ is called the latent hardening modulus. Generally speaking, $\chi$ varies during plastic deformation. As $\chi$ increases, the vertex effect weakens. If $\chi=0$, very sharp vertexes will ap$\operatorname{pear}^{[8]}$.

The resolved shear stress rate of an active slip system should equal the sum of the latent hardening rate $\dot{\boldsymbol{\tau}}_{\text {active }}$ and the active hardening rate $\dot{\boldsymbol{\tau}}_{\text {active, so }}$

$$
t=t_{\text {active }}+t_{\text {latent }}=h \dot{\gamma}+\chi \boldsymbol{P}: \boldsymbol{D}^{\mathrm{p}},
$$

where $h$ is the active hardening modulus. Obviously, when $h=0$, eq. (8) becomes the expression of Budiansky and Wu's theory ${ }^{[9]}$.

An active slip system may slide in $\boldsymbol{m}$ or the reverse direction. If the slip systems comply with Prager's kinematic hardening rule, $\tau_{+\mathrm{cr}}-\tau_{-\mathrm{cr}}$ remains constant. $\tau_{+\mathrm{cr}}-\tau_{-\mathrm{cr}}$ normally varies more or less during deformation, so an attendant Bauschinger coefficient $\beta=\left(\tau_{+\mathrm{cr}}-\tau_{-\mathrm{cr}}\right) / 2 \tau_{\mathrm{cr} 0}$ is introduced in the present work. Here, $\tau_{\mathrm{cr}}$ is the initial critical resolved shear stress. The activation state of a slip system can be described as

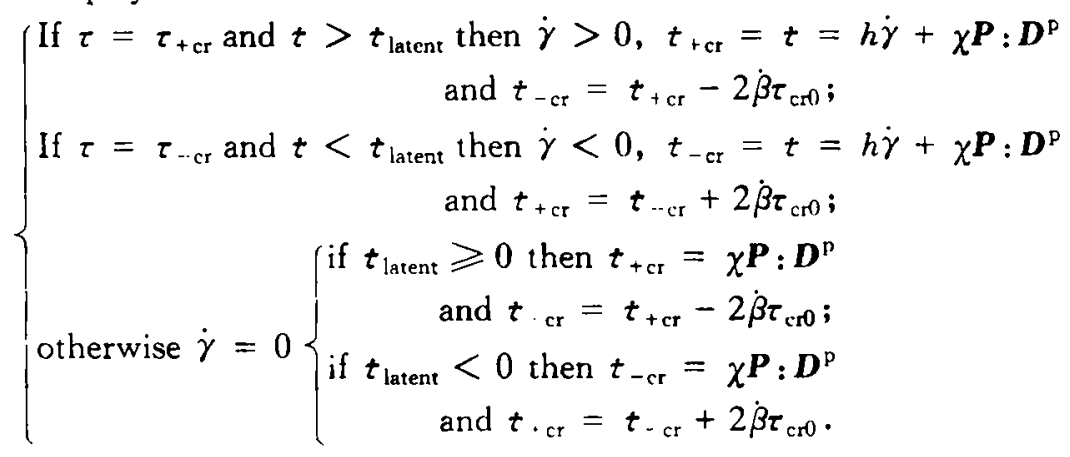

Eq. (9) enables the subsequent surfaces to possess all of the three characteristics mentioned above.

Compared with Prager's kinematic hardening rule, eq. (7) means that the latent hardening rate can also be calculated from a back-stress rate tensor $\hat{\boldsymbol{T}}_{\text {back }}$ proportional to the plastic strain rate $D^{\text {p }}$, i.e.

$$
\hat{\boldsymbol{T}}_{\text {back }}=\lambda \boldsymbol{D}^{\mathrm{p}} \text { and } \boldsymbol{t}_{\text {latent }}^{(a)}=\boldsymbol{P}^{(a)}: \hat{\boldsymbol{T}}_{\text {back}} .
$$

Suppose that the finite deformation effect is negligible and the plastic deformation does not affect the elastic property of material. Then

$$
t=\boldsymbol{P}: \dot{\boldsymbol{T}}
$$

and

$$
D^{\mathrm{p}}=D-D^{\mathrm{e}}=D-C^{\mathbf{e}}: \boldsymbol{T},
$$

where $\boldsymbol{D}$ is the overall strain rate tensor and $\boldsymbol{C}^{e}$, the elastic compliance tensor. Denote the 4-order unit tensor with $I D I$ and consider eqs. (6), (8), (11) and (12). Then

$$
\left[\boldsymbol{I} \bowtie \boldsymbol{I}+\int_{\Omega} \int_{\Psi} \frac{\chi}{h} \boldsymbol{P} \otimes \boldsymbol{P} \mathrm{d} \Omega \mathrm{d} \Psi\right]: \boldsymbol{D}
$$




$$
=\left[\boldsymbol{C}^{\mathbf{e}}+\int_{\Omega} \int_{\Psi} \frac{1}{h}\left[\boldsymbol{P} \otimes \boldsymbol{P}+\chi^{P} \otimes \boldsymbol{P}: \boldsymbol{C}^{\mathrm{e}}\right] \mathrm{d} \boldsymbol{\Omega} \mathrm{d} \Psi\right]: \boldsymbol{T} .
$$

$\mathrm{Eq}$. (13) is an explicit expression of the constitutive equation with two kinds of material parameters $h$ and $\chi$. $\beta$ plays an important role in determining when a slip system will be reactivated in the reverse direction after an unloading process. The elastoplastic stiffness or compliance tensor can be obtained with eq. (13). However, in a new loading step, the current values of $h$ and $\chi$ are not known because they depend on the deformation history and the activation states of slip systems. It is not always convenient to use eq. (13) in numerical analysis. An alternative method to calculate the stress-strain responses of material is as follows.

Substituting eqs. (6) and (11) into eq. (8), we get

$$
\dot{\gamma}=\frac{\boldsymbol{P}: \dot{\boldsymbol{T}}}{h}-\frac{\chi}{h} \int_{\Omega} \int_{\Psi}(\boldsymbol{P}: \tilde{\boldsymbol{P}}) \tilde{\gamma} \mathrm{d} \Omega \mathrm{d} \Psi,
$$

where $\dot{\gamma}$ mean that the corresponding variable is a function of the integral variable. The above equation is a standard Fredholm integral equation with a symmetric integral kernel $\boldsymbol{P}: \tilde{\boldsymbol{P}}=\tilde{\boldsymbol{P}}: \boldsymbol{P}$. If $h>0$, eq. (14) has one and only one solution ${ }^{10 !}$. By dividing half of the spherical angle $2 \pi$ (arientation range of $\boldsymbol{n}$ ) into $N$ equal parts, and half of the plane angle $\pi$ (orientation range of $\boldsymbol{m}$ ) into $M$ equal parts, a discrete model with $M \times N$ slip systems will be formed. In this case, the integration in eqs. (6), (13) and (14) will also be replaced by summation. Particularly, eq. (14) becomes

$$
\dot{\gamma}^{(j)}=\frac{1}{h^{(j)}} \boldsymbol{P}^{(j)}: \dot{\boldsymbol{T}}-\frac{2 \pi^{2}}{M N} \frac{\chi}{h^{(j)}} \sum_{i=1}^{M N} \dot{\gamma}^{(i)}\left(\boldsymbol{P}^{(j)}: \boldsymbol{P}^{(i)}\right)
$$

(for all of the active slip systems).

This is a set of algebraical equations with a symmetric and positive-definite coefficient matrix. It is not difficult to find $\dot{\gamma}^{(j)}$ from eq. (15), hence the strain rate tensor from equations (6) and (12).

\section{Influence of material property parameters on subsequent yield surfaces}

Since the basic component of the present material model is simply a slip system with only one degree of freedom, it is not difficult to formulate the evolution patterns of $h, \chi$ and $\beta$. For a certain material, the material constants in the evolution equations can be calibrated through comparison of the theoretical predictions with experimental results ${ }^{[1]}$.

The fundamental features of the present material model will be shown using numerical examples in which $h, \chi$ and $\beta$ are provided with typical evolution patterns, even constants.

Two sets of simulated tensile stress-strain curves with $h$ and $\chi$ being constants are plotted in fig. 1(a) and (b). Obviously the present material model can well simulate materials with arbitrary strain hardening property. $\beta$ does not affect the stress-strain responses of material under monotonically proportional loading conditions.

If $\chi=0$ and $\beta=1$, the present material model degenerates into the simple slip model proposed by Batdorf and Budiansky ${ }^{[3,12]}$. When the latent hardening effect is taken into account, i.e. $\chi>0$, the activation states of slip systems differ from the prediction given by Batdorf and Budiansky ${ }^{[3,12]}$. The orientation distribution of sliding lines in polycrystalline aluminum under tension was measured by Johnson and Batdorf ${ }^{[13]}$. Fig. 2(a) shows the comparison of some theoretical 

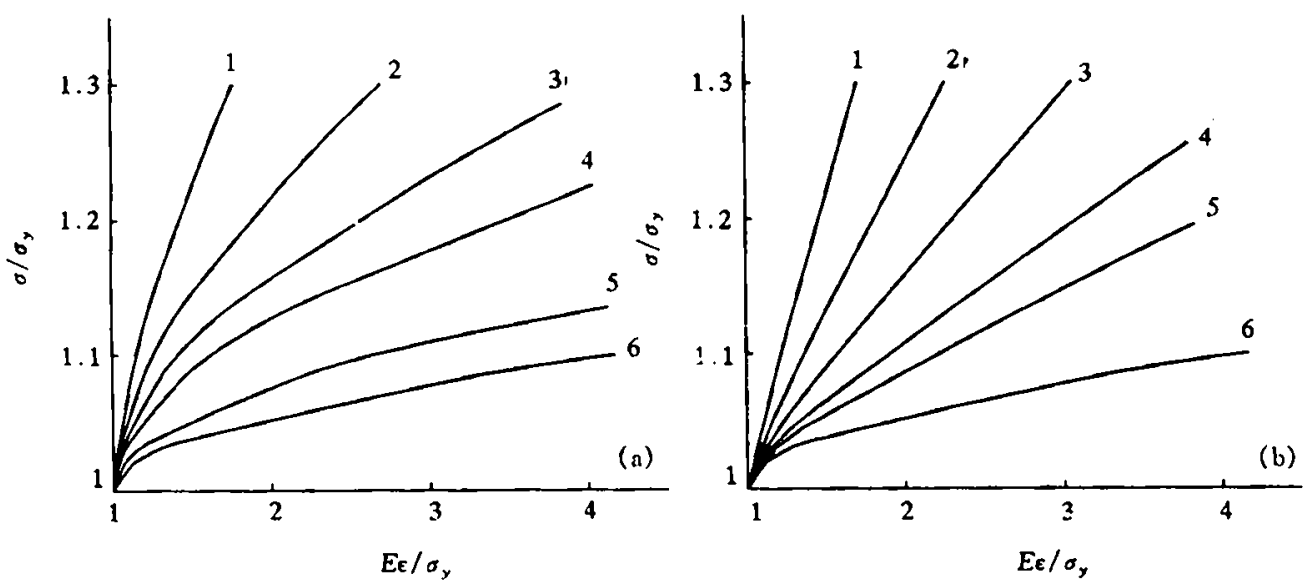

Fig. 1. (a) Simulated tensile stress-strain curves with $\chi=0.1, h=0.2 E ; 2, h=0.1 E ; 3, h=0.05 E ; 4, h$ $=0.03 E ; 5, h=0.01 E ; 6, h=0.005 E$. (b) Simulated tensile stress-strain curves with $h=0.005 E .1, \chi / h=40$;

$2, \chi / h=30 ; 3, \chi / h=20 ; 4, \chi / h=10 ; 5, \chi / h=6 ; 6, \chi / h=0$.

predictions with the experiments. The stress-strain curve in fig. 2 (b) is well simulated by the present material model with material constants $\sigma_{y}=4.3 \mathrm{ksi}, E=2150 \mathrm{ksi}, h=60.63 \exp$ $(-30.50 \gamma)+3.87$ and $\chi=4.0\left(\sum\left|\dot{\gamma}_{i} h_{i}\right| / \sum\left|\dot{\gamma}_{i}\right|\right)$. Obviously, the prediction of the present material model is almost coincident with the experimental results. If the latent hardening effect is ignored, the orientation range of activated slip systems will be $15^{\circ}-75^{\circ}$ when the tensile stress raises to twice the initial yield stress. Actually, latent hardening causes the critical resolved shear stress of the slip systems to increase during deformation, so the orientation range is reduced approximately to $30^{\circ}-60^{\circ}$.
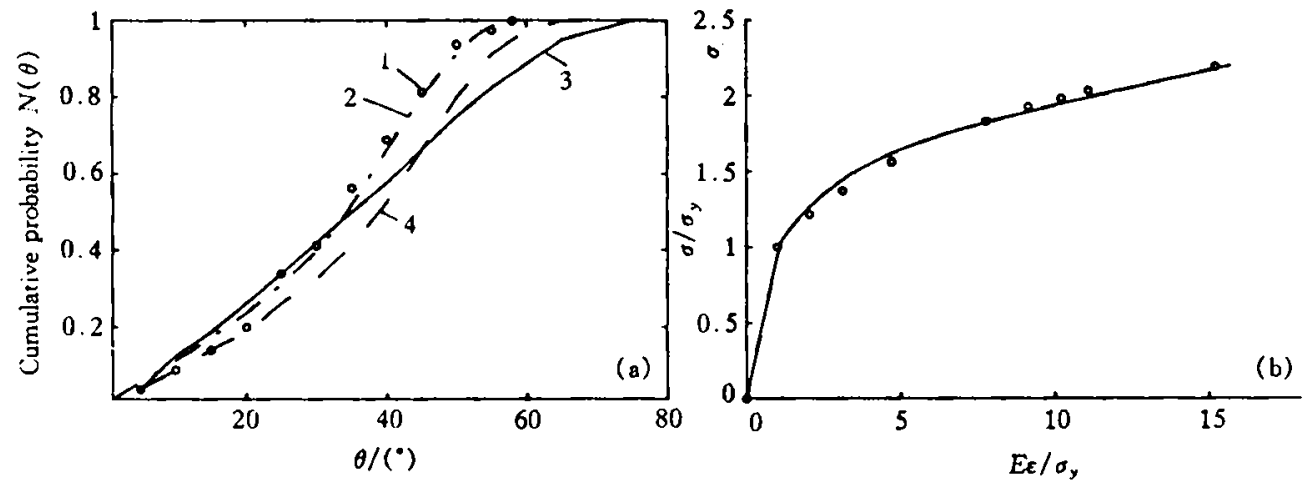

Fig. 2. (a) Predicted and measured slip-angle distribution $\left(\sigma / \sigma_{r}=2\right) .1$, Experiment; 2, present study; 3, single slip mode by ref. [3]; 4 , simple slip mode with FCC by reference[3]. (b) Simulated tensile stress-strain curve of 2S-0 aluminum alloy. $O$, Experimental point; - , simulated curve.

Figure 3(a) illustrates the effect of $\chi$. (i) Curvatrue of the subsequent yield surfaces at a prestress point decreases as $\chi$ increases. It almost remains unchanged when $\chi$ is large enough, but a sharp corner will appear when $\chi=0$. (ii) The size of the subsequent yield surfaces in the direction perpendicular to the prestress path decreases when $\chi=0$, but it will remain unchanged when $\chi$ is large enough. 
Figure 3(b) illustrates the effect of $\beta$. Size of the subsequent yield surfaces in the direction along the prestress path reduces as $\beta$ increases. When $\beta=1$, the size remains unchanged.

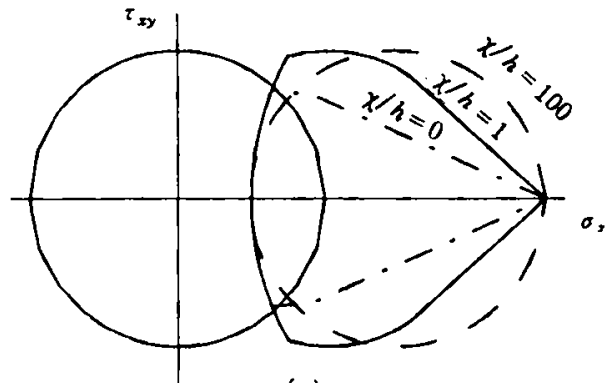

(a)

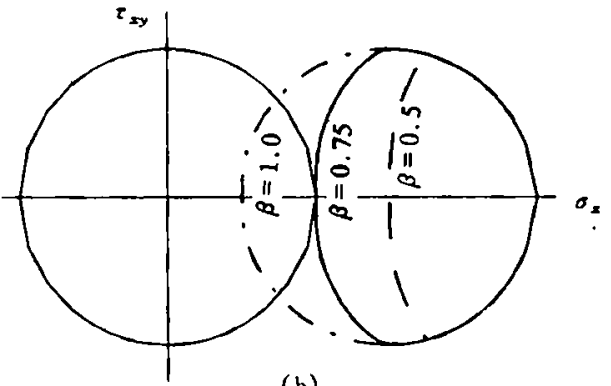

(b)

Fig. 3. (a) Vertex and cross effects. (b) Bauschinger effect $\chi / h=15$.

Some of the simulated subsequent yield surfaces are plotted in fig. 4. They are favorably
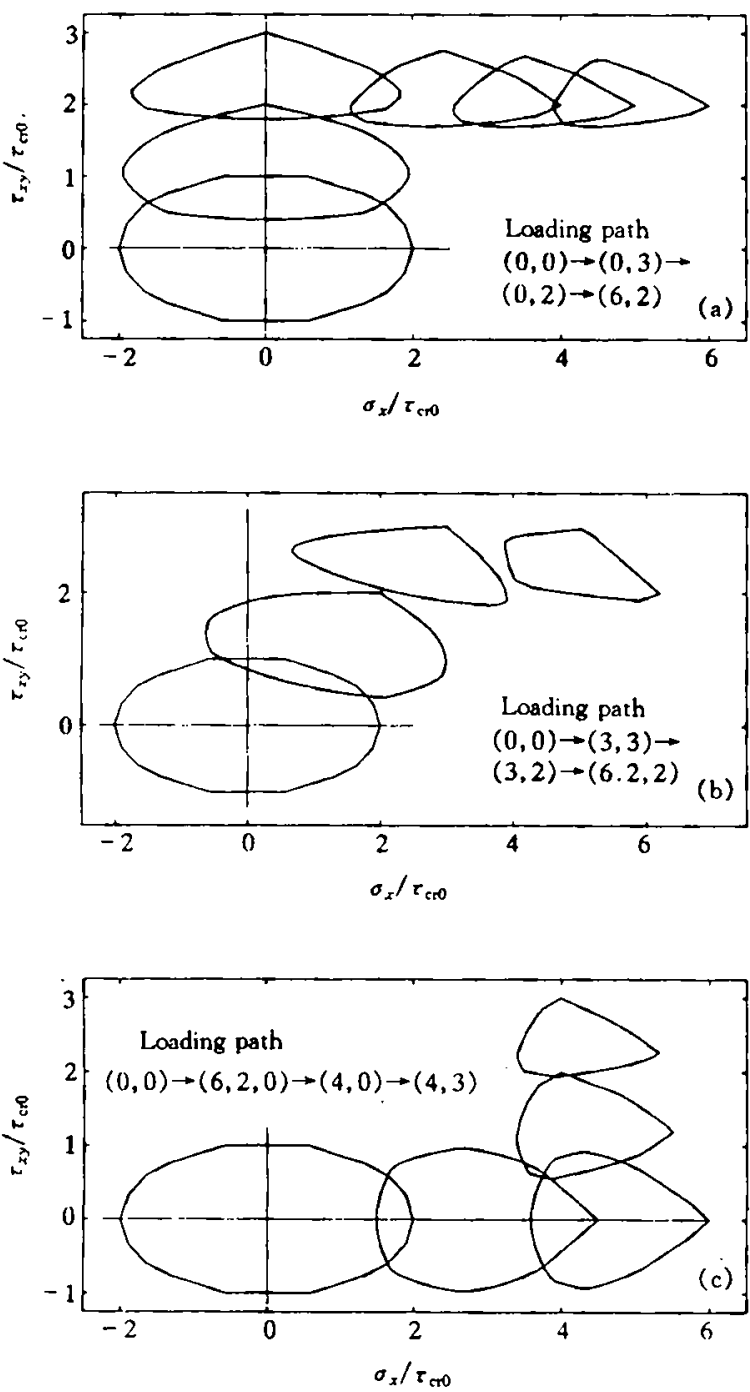

Fig. 4. Evolution of simulated subsequent yield surfaces. compared with the experiments ${ }^{[6]}$. The slight discrepancy may be further reduced by taking the viscosity effect into account.

\section{Fundamental features of the present materi- al model}

(1) Different from the phenomenological plasticity theories, the present material model requires neither plasticity potential functions nor assumed evolution rules of thermodynamic intrinsic variables. As long as $h, \chi$ and $\beta$ are available, it is capable of predicting the macroelastoplastic behavior of material under complex loading conditions.

(2) Basic components of the present material model are the equivalent slip systems, each of which has only one deformation degree of freedom. The overall stress-strain responses of material can be obtained by means of component-wise calculation. The present material model is efficient for numerical analysis.

(3) For plane stress problems, it can be easily verified that the equivalent stress-strain curves of material under radial loading conditions are exactly identical when the elastic strain and hydrostatic stress are ignored. In this case, the material is actually in pure shear states in respective principal directions. Because of the orientation symmetry of the slip systems, the $u$ niqueness of equivalent stress-strain curves will 
be naturally guaranteed. In the phenomenological plasticity theory, this uniqueness is only a postulate $^{[4]}$. Numerical results obtained with the present material model indicate that the uniqueness postulate is also a good approximation under arbitrary radial loading conditions.

(4) Theoretically speaking, activation of a slip system means commencement of plastic defor-
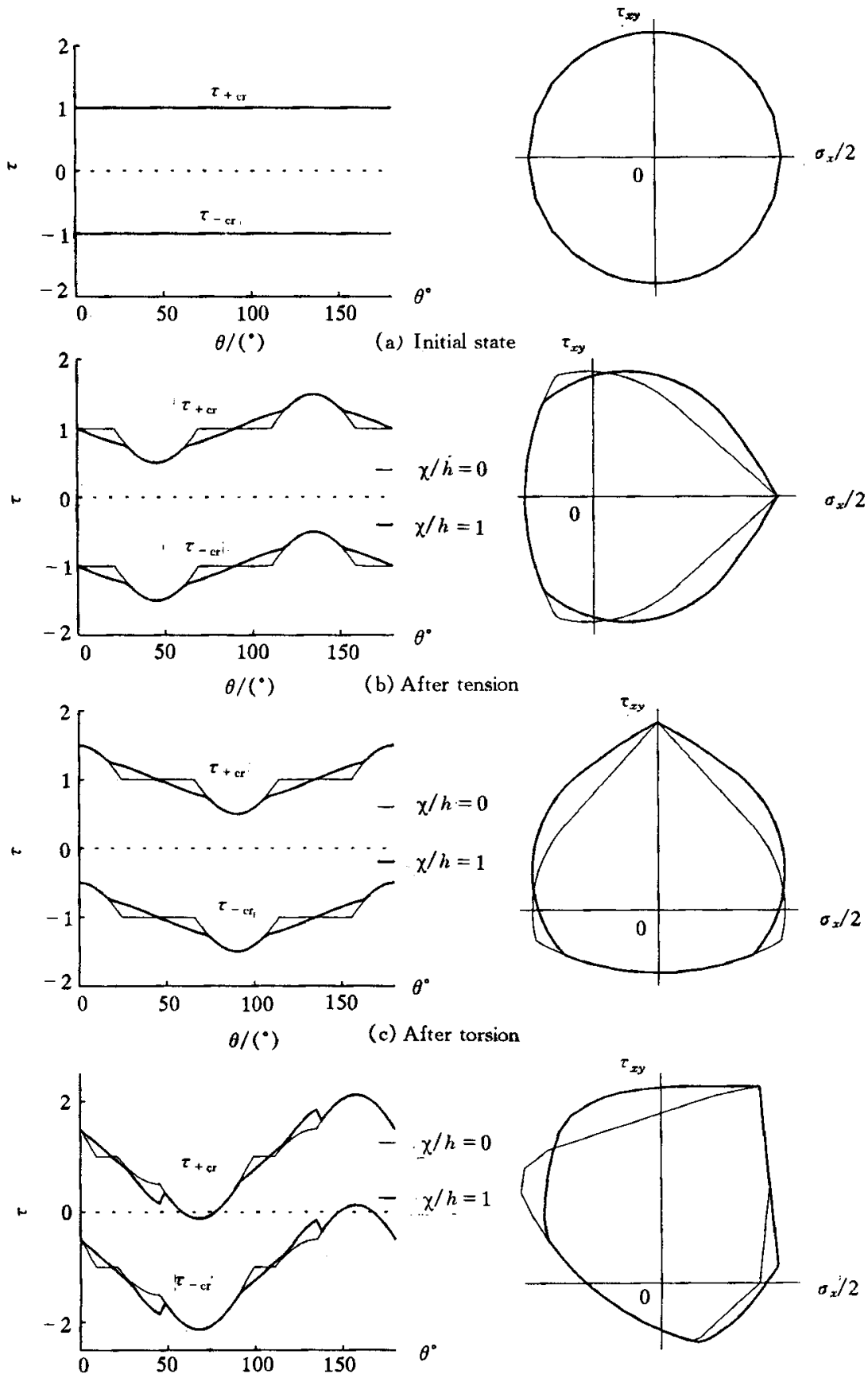

(d) After tension- torsion

Fig. 5. Distribution of $\tau_{ \pm \mathrm{cr}}$ and the corresponding yield surfaces. (a) Initial state; (b) after tension: (c) after torsion; (d) after tension-torsion 
mation. A macroscopic yield point should be reached if one of the slip systems attains to its critical state, i.e.

$$
\boldsymbol{P}: \boldsymbol{T}=\tau_{ \pm \mathrm{cr}} .
$$

In the 6-D stress space, $\boldsymbol{P}$ is a vector of a constant magnitude and $\boldsymbol{P}: \boldsymbol{T}$ is the projection of $\boldsymbol{T}$ on $\boldsymbol{P}$. Eq. (16) expresses two hyperplanes confining the stress range, in which the slip system remains static ${ }^{[4]}$. The internal envelope hypersurface of the hyperplanes corresponding to all of the slip systems will be a macro-yield surface. Obviously, the yield surfaces are certainly convex and can be determined with $\tau_{ \pm \mathrm{cr}}$ at any time. For convenience, a 2-D material model with both $n$ and $\boldsymbol{m}$ in the $X-Y$ plane is taken as an example. $\tau_{ \pm \mathrm{cr}}$ as a function of the angle between $\boldsymbol{m}$ and the $X$ axis is plotted on the left side of fig. 5. The corresponding yield surfaces are shown in the right side. In this sense, $\tau_{ \pm \text {cr }}$ can be regarded as intrinsic variables and eq. (9) is their evolution rule.

(5) In eq. (9), $\tau_{+c r}$ and $\tau_{-c r}$ are related by the attendant Bauschinger coefficient, so the present material model has realized the presage of $\mathrm{Wang}^{[14]}$, improving the plasticity theory of linear loading functions developed by Sanders et al. ${ }^{[15]}$. If the maximum shear stress of slip systems is constant, there must exist a limit yield surface, which affords a physical meaning to Mroz's ${ }^{[16]}$ multi-yield surface theory.

(6) As long as the active hardening modulus $h>0$, the present material model has all of the mechanical properties drawn from Drucker's postulate. In this sense, a plasticity potential function has been determined by the present material model. A good prediction of subsequent yield surfaces will also be reliable evidence of the rationality and accuracy of the present material model.
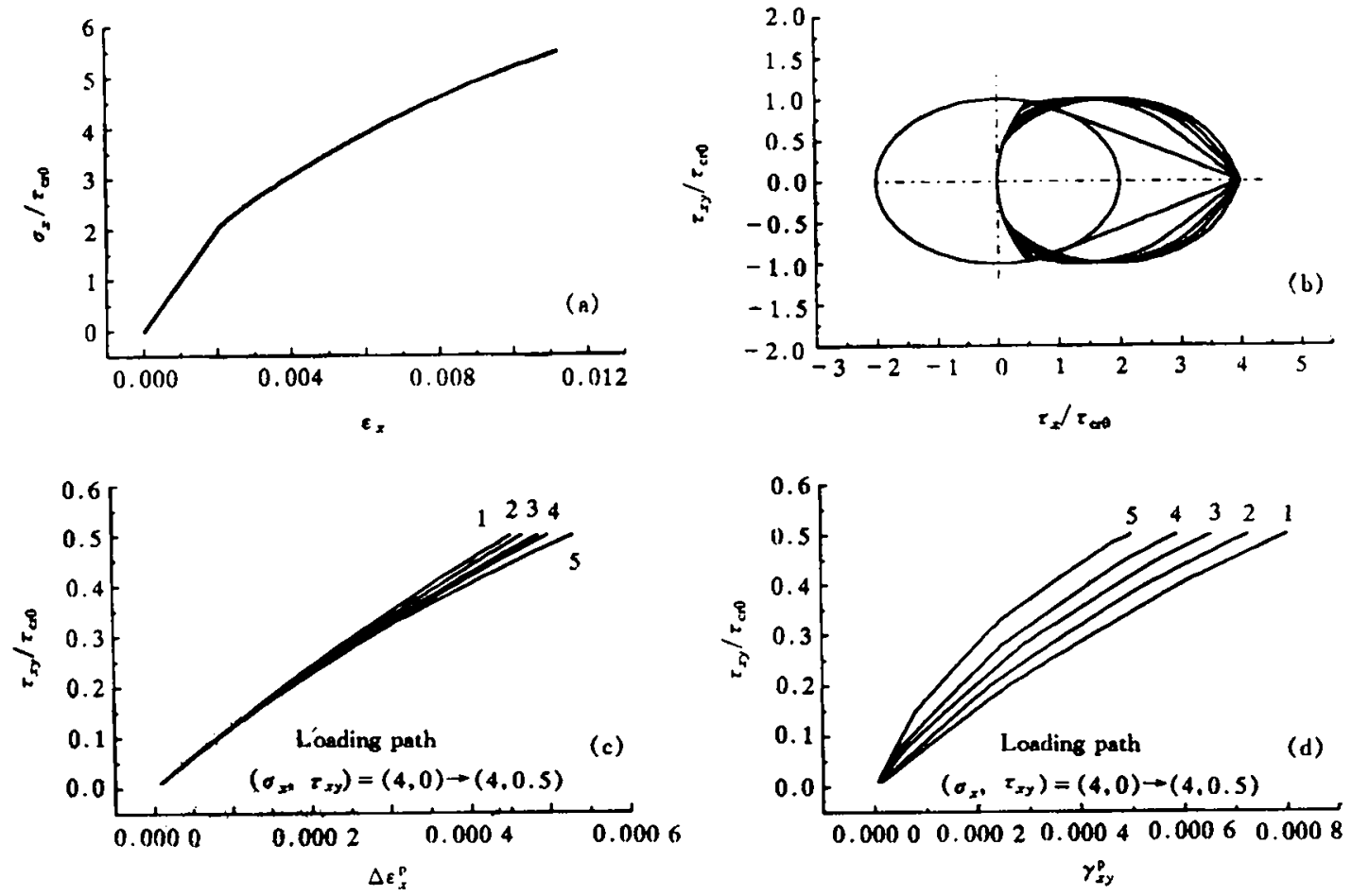

Fig. 6. Subsequent yield surfaces, tensile and shear plastic strains during torsion. Lines $1-5$ correspond to $\chi / h$ $=0.1,2.5,5,15$. (a) Simulated $\sigma_{x}-\varepsilon_{x}$ curve; (b) subsequent yield surfaces; (c) tensile plastic strain; (d) shear plastic strain. 
For example, a uniaxial stress-strain curve in fig. 6(a) is well simulated with any of the five combinations of $h$ and $\chi$, but the subsequent yield surfaces shown in fig. 6(b) are different. If $\chi / h$ $=0$, there is a sharp corner at the loading point, as predicted by the simple slip theory ${ }^{[3,12]}$. When $\chi / h$ is large enough, the shape of the subsequent yield surfaces remains almost unchanged, as predicted by Prager's theory. Fig. 6(b) implies that when the shear stress is applied with tensile stress remaining, the strain responses predicted by the present material model depend on $\chi /$ $h$. The tensile and the shear plastic strain during torsion are plotted in figs. 6(c) and (d), respectively. These figures indicate that the appropriate $\chi / h$ of a certain material has to be determined through nonradial loading tests.

The evolution pattern of $h, \chi$ and $\beta$ for commonly-used metals and the material constant calibration method are reported elsewhere ${ }^{[11.17]}$.

\section{References}

1 Havner, K. S., Finite Plastic Deformation of Crystalline Solids, New York: Cambridge University Press, 1992, Chap. 3.

2 Tokuda, M., Kratochvil, J. Ohashi, Y., Mechanism of induced plastic anisotropy on polycrystalline metals, Phys. Stat . Sol., Ser. A, 1981, 68: 629 .

3 Batdorf, S. B., Budiansky, B., A mathematical theory of plasticity based on the concept of slip, NACA TN, 1994. 1871.

4 Martin, J. B., Plasticity: Fundationals and General Results, The MIT Press, 1975, Chap. 7.

5 Liang, V. G., Cheng, P. S., A constitutive model of elasto-plastic materials based on fibre-reinforcing and sliding mechanisms, Science in China, Ser. A, 1993, 36: 692.

6 Philips, A., Tang, J. L., The effect of loading path on the yield surface at elevated temperatures, Int. J. Solids Structure, $1972,8: 463$.

7 Lin, T. H., Ito, Y. M., Theoretical plastic stress-strain relationship of a polycrystal and comparisons with Von Mises' and Tresca's plasticity theories, Int. J. Eng. Sci., 1966, 4:543.

8 Hutchinson, J. W., Elastic-plastic behavior of polycrystalline metals and composites, Proc. Roy Soc., U. K. London, Ser. A, 1970, 319: 247

9 Budiansky, B., Wu, T. T., Experimental studies of polyaxial stress-strain laws of plasticity, Proc. 4th U.S. Nat . Congr. Appl. Mech., ASME, New York: Pergamon Press, 1962, 1175.

10 Delves, L. M., Mohamed, J. I.., Computational Method for Integral Equations, Cambridge: Cambridge University Press, 1985, Chaps. 3, 4.

11 Liang, N. G., Xu, T., Wang, Z. Q., A new expression of hardening coefficients for fcc-crystal and calibration of the material constants, Science in China, Ser. A, 1995, 38: 945

12 Batdorf, S. B., Budiansky, B., Polyaxial stress-strain relations of a strain-hardening metal, J. Appl. Mech., 1954, 11: 323.

13 Johnson, A. E., Batdorf, S. B., A study of slip formation in polycrystalline alluminum, NACA TN2576, 1951.

14 Wang, R., Xiong, Z. H., Huang, W. B., Foundation of Plasticity (in Chinese), Beijing: Science Press, 1982, Chap. 4.

15 Sanders, J. L., Jr., Plastic stress-strain relations based on linear loading functions, Proc. 2 nd U.S. Nat. Congr. Appl. Mech., New York: SME, 1954, 455 .

16 Mroz, Z., On the description of anistropic work hardening, J. Mech. Phys. Sol., 1967, 15: 163.

17 Liu, H. Q. . Liang, N. G. . Mechanical-property parameters of polycrystalline metal and prediction of stress-strain responses under out-of phase circular deformation, Proc. 1 st Asia-Ocenia Int. Sym. On Plasticity, Beijing: International Academic Publishers, 1993. 438. 